\title{
Prolonged Sleep-Onset Latency during Chronic Sleep Restriction in Mice
}

\author{
Bowon Kim, BA ${ }^{1,2}$, Eunjin Hwang, $\mathrm{PhD}^{1}$, Youngsoo Kim, $\mathrm{PhD}^{3}$, Jee Hyun Choi, $\mathrm{PhD}^{1,4}$ \\ ${ }^{1}$ Center for Neuroscience, Korea Institute of Science and Technology, Seoul, Korea \\ 2Department of Medical Science, College of Medicine, Yonsei University, Seoul, Korea \\ ${ }^{3}$ Department of Psychiatry, VA Boston Healthcare System, Research Service and Harvard Medical School, Brockton, MA, USA \\ ${ }^{4}$ Department of Neuroscience, University of Science and Technology, Daejeon, Korea
}

\begin{abstract}
Background and Objective Even though we know that sleep is important for maintaining normal cognitive and behavioral functions, it is still unclear whether sustained sleep restriction, which is prevalent in modern society, is adaptive or whether it leads to a functionally and physiologically deteriorated state. On the assumption that sleep-onset latency (SOL) and sleep pressure are inversely correlated, we hypothesized that an adaptation to chronic sleep restriction (CSR) might pay off cumulative sleep pressure and thus we investigated the influence of CSR by measuring SOL as a function of sleep restriction day.

Methods Mice $(n=9)$ were sleep deprived for $18 \mathrm{~h}$ a day using periodically rotating wheels. This was followed by a $6 \mathrm{~h}$ period of sleep opportunity (SO) that started at the beginning of each light period. This sleep restriction protocol was repeated for 5 consecutive days. SOL was defined as the duration of wake state from light-on time to first non-rapid eye movement epoch. Total wake time during SO was calculated following sleep restriction and during the same period on baseline and during the first recovery day.

Results SOL gradually increased from the first day of sleep restriction onward (paired t-test; $\mathrm{p}<0.01$ for SR 1 vs. SR5 and $\mathrm{p}<0.05$ for SR3 vs. SR5). The linear regression slope of SOL as the function of SR days in individual mice showed a significantly positive drift (average $\mathrm{R}$-squared $=0.69$, average slope $=8.1 \mathrm{~min} /$ day) .

Conclusions In the present study, sleep onset was delayed in sleep restriction day compared to the baseline sleep and SOL increased across chronic sleep restriction days in mice. This finding is different from our expectation that SOL will decrease compared to baseline sleep and then return to baseline level as CSR continues. Instead, SOL increased gradually as a function of sleep restriction days. This indicates that SOL may not simply reflect sleep pressure, rejecting our assumed inverse correlation between SOL and sleep pressure.
\end{abstract}

Sleep Med Res 2013;4(1):28-32

Key Words Sleep, Chronic sleep restriction, SOL, Mice.

Received: September 6, 2014

Revised: October 15, 2014

Accepted: October 22, 2014

Correspondence

Jee Hyun Choi, $\mathrm{PhD}$

Center for Neuroscience, Korea Institute of

Science and Technology, 5 Hwarang-ro

14-gil, Seongbuk-gu, Seoul 136-791, Korea

Tel $+82-2-958-6952$

Fax +82-2-958-6737

E-mail_jeechoi@kist.re.kr

\section{INTRODUCTION}

Our knowledge of the importance of sleep for maintaining normal cognitive, physiological, and behavioral functions continues to improve. ${ }^{1-5}$ However, in the 24-7 treadmill of modern life, many of us are chronically sleep deprived due to extended working hours or study or unceasing internet network services. According to the National Sleep Foundation, there has been no decline in sleep time since 1965 (American Time Use Survey). ${ }^{6}$ As cumulative hours of sleep loss are defined as sleep debt, ${ }^{7}$ the consequences of this built-up sleep debt are not yet known. In a systematic and controlled study, Horne and Wilkinson ${ }^{8}$ reported that there were no significant differences in performance between half-night sleep reduction group and all-night sleep group. Likewise, Youngstedt et al. ${ }^{9}$ observed no functional changes such as mood changes or sleepiness nor any physiological alterations in parameters such as glucose tolerance or insulin sensitivity. ${ }^{10}$ However, Van Dongen et al..$^{5}$ reported that neurobehavioral functions deteriorated with sleep debt in a dose-dependent way and a recently reviewed biochemical study suggested a link between persistent sleep debt and impairment of protein synthesis and synaptic plasticity. ${ }^{11}$ Taken together, it is still not clear whether sustained sleep restriction can be adaptive or whether it 
leads to a functionally/physiologically deteriorated state.

In this study, we investigate the effect of chronic sleep restriction (CSR) by measuring the sleep onset latency (SOL) as a function of sleep restriction day. In numerous human studies, SOL has been successfully used to evaluate the sleep debt as an objective scale for sleepiness. ${ }^{12,13}$ On the assumption that SOL and sleep pressure are inversely correlated, we predict that if chronic sleep restriction is adaptive, the sleep debt will decrease at a certain moment and SOL will rebound. But if chronic sleep restriction leads to a persistently impaired state, the sleep debt will not be ameliorated by an adaptation process, and therefore SOL will not recover to baseline. Belenky et al., in a study of humans, observed no rebound of SOL during 7 days of chronic sleep restriction, which was accompanied by a continuous decrease of performance in a dose-dependent way. However, in rats, Kim et al. ${ }^{14}$ reported a rebound of SOL at day 5 of chronic sleep restriction. Comparing both species, rats are known to die within a month of sleep deprivation, ${ }^{15}$ whereas human tolerance is longer, up to six months. ${ }^{16}$ Therefore, we anticipate that rodents behave according to a faster clock than that of humans. Here, we applied a chronic sleep restriction paradigm in mice, known to have a shorter life span than rats and to be more vulnerable to sleep deprivation. If the rebound of SOL at day 5 of chronic sleep restriction in rats represents the adaptation to sleep loss, we would expect to see a faster or similar rebound in mice.

\section{METHODS}

\section{Animals and Surgery}

Male C57BL/6 and 129S4/SvJae hybrid F1 mice (12 \pm 1 weeks, $\mathrm{n}=9$ ) were used. Animals were maintained on a 12:12-h light:dark cycle, with the light going on at 8 am and they were given ad libitum access to food and water. Surgery protocols were approved by the Institutional Animal Care and Use Committee at Korea Institute of Science and Technology. Animals were implanted with three screw electrodes under ketamine and xylazine anesthesia (120 and $6 \mathrm{mg} / \mathrm{kg}$, respectively). The location of screw electrodes was frontal [anterior-posterior (AP), 1.5; medial-lateral (ML), -2 mm from bregma), parietal (AP, -2; ML, 4 $\mathrm{mm}$ from bregma) area, and cerebellum (-6; ML, $-2 \mathrm{~mm}$ from bregma) for reference. After surgery, all electrodes were secured permanently to the skull using dental cement with two to three additional anchoring screws implanted into the skull.

\section{Chronic Sleep Restriction Paradigm}

After a recovery from the surgery, animals were habituated to experimental settings with a new light-on schedule (9 am or 1 $\mathrm{pm}$ ) for at least 5 days. Prior to the experiment, the mice were exposed to the new light-on system for the same number of days of the time difference in hours (i.e., 1 and 5 days for 9 am and 1 pm groups, respectively), and during these days, the mice were put into the sleep restriction cage with a motorized wheel for two hours in each day for the animals to be habituated to the intervention of sleep. During experiments, mice inhabited a cylindrical acrylic cage (7.8 inch in diameter) in a light- and sound-proof behavior box. In the same box, behind the cage, an automatic wheel (5.5 inch in diameter $\times 2.3$ inch in width) was located for sleep restriction. Screw electroencephalographies (EEGs) were recorded over a $24 \mathrm{~h}$ period for the whole day using a Grass amplifier (QP511, Grass Technologies, Warwick, RI, USA) with a $500 \mathrm{~Hz}$ sampling rate, a high-pass filter at $0.3 \mathrm{~Hz}$, a low-pass filter at $100 \mathrm{~Hz}$, and a $60 \mathrm{~Hz}$ built-in notch filter. After $24 \mathrm{~h}$ of recording of baseline, mice were moved to the wheel for sleep restriction for $18 \mathrm{~h}$ a day with a " $4 \mathrm{~s}$ on- $2 \mathrm{~s}$ off" schedule at a speed of approximately 2.3 RPM. Following sleep restriction, mice were moved back into their cage, giving them a sleep opportunity $(\mathrm{SO})$ of $6 \mathrm{~h}$ per day. After this the next day of sleep restriction started and this schedule was repeated for 5 days (SR1-5). After 5 days of sleep restriction, mice had additional days in the cage without disruption in order to recover. Zeitgeber time (ZT) was used as a convenient comparison between mice on different time schedules, with light-on time indicated as ZT0 (Fig. 1).

\section{Sleep Scoring}

Sleep stages were manually scored by sleep experts, using SleepSign, a sleep analysis software program (Fig. 2). Based on the properties of screw EEGs in both frontal and parietal, and accelerometer signal, the total signals were divided into 10-s epochs and scored as one of three states: non-rapid eye movement (NREM) sleep, rapid eye movement (REM) sleep, and wake states. SOL was defined as the time elapsed between the onset of the SO period (ZT0) and the first episode of NREM sleep. Without any disturbance to mice on baseline and the recovery day, animals spontaneously stayed awake at the time of light-on. Total wake time (TWT) during SO was calculated following $18 \mathrm{~h}$ of sleep restriction and during the same period on baseline and the recovery day 1 .

\section{RESULTS}

As shown in Fig. 3A, SOL gradually increased from the first day of sleep restriction [Kruskal-Wallis analysis of variance (ANOVA) test, $\mathrm{p}=0.002$; post-hoc paired t-test with Bonferroni correction, $\mathrm{p}<0.05$ for SR day 1 (SR1) vs. baseline, $\mathrm{p}<0.01$ for $\mathrm{SR} 3$ vs. baseline and $\mathrm{p}<0.01$ for SR5 vs. baseline]. Furthermore, SOL on SR5 is enhanced compared to SR 1 and 3 ( $p<0.01$ for $\mathrm{SR} 1$ vs. SR5 and $\mathrm{p}<0.05$ for SR3 vs. SR5). The linear regression slope of SOL as the function of SR days in individual mice showed a significantly positive drift (average R-squared $=0.69$, average slope $=8.1 \mathrm{~min} /$ day). In addition, compared to the baseline, SOL increased by $1482 \pm 1213 \%$ (mean \pm standard error mean), $833 \pm 492 \%$, and $1832 \pm 936 \%$ on SR1, SR3 and SR5, re- 
spectively. Although SOL was significantly increased, the range of the increment varied between individuals. In particular, on SR5, the latency increment ranged from $127 \%$ to $8833 \%$ in all mice. This gradual increase of SOL over 5 days of sleep restriction returned to baseline level on the first day of recovery.

The change in SOL was comparable with the change in TWT. TWT was also gradually increased over 5 days of sleep restriction (Table 1). The amount of time spent awake after sleep has been initiated and before final awakening, defined as the difference between TWT and SOL, showed no significant difference from baseline on SR1, whereas the amount of wake time on SR3 and recovery showed a slightly increasing trend (Fig. 3B) (Kruskal-Wallis ANOVA test, $\mathrm{p}=0.03$; post-hoc paired t-test with Bonferroni correction, not significant for baseline vs. SR1 and SR5, $\mathrm{p}<0.05$ for SR3 vs. baseline, and $\mathrm{p}<0.5$ for SR1 vs. SR3 and SR5). This finding indicates that the increased TWT during sleep opportunity is accounted for by the increased SOL in the case of the early period of sleep restriction.

Additionally, we investigated correlation between SOL and frontal delta power, which is electrophysiological maker of sleep pressure (data was not shown). In accordance to our assumption that sleep pressure increases through the days of CSR, SOL and frontal delta power is altered during CSR. Likewise, there is significant correlation between SOL and frontal delta power in whole samples $[\mathrm{n}=54$ ( 9 animals $\times 6$ days), $\mathrm{r}=0.51, \mathrm{p}<0.001$, Pearson's linear correlation]. However, when we calculated correlation values on each day $(n=9)$, none of the days had signifi- cant correlation between SOL and frontal delta power.

\section{DISCUSSION}

In the present study, CSR significantly altered SOL in mice. We expected SOL to decrease and then return to baseline level as CSR continues, but instead, the SOL was higher than SOL of normal sleep and this step-up of SOL increased gradually as a function of sleep restriction days. This result indicates that SOL may not simply reflect sleep pressure, rejecting the assumption of the inverse correlation between SOL and sleep pressure.

As mentioned in the introduction, SOL has been used to indicate how much sleep is needed. In a multiple sleep latency test, a lower sleep latency would indicate severe sleepiness. ${ }^{13}$ Belenky et al. ${ }^{4}$ and Van Dongen et al. ${ }^{5}$ supported SOL as a sleep pressure marker with evidence that the SOL decreased abruptly on SR1 and remained at the level of SR2 in the subsequent 7 days of CSR. In their study, the latency on SR1 (acute sleep restriction) was half that of the of baseline value. However, in our mice study, SOL started to increase from the first day of sleep restriction.

In previous acute sleep deprivation studies in rodents, increased SOL was observed as well, ${ }^{17,18}$ but was interpreted as the result of stress rather than sleep deprivation. In a less stressful sleep restriction study of rats, SOL decreased. ${ }^{14}$ We used the lat-

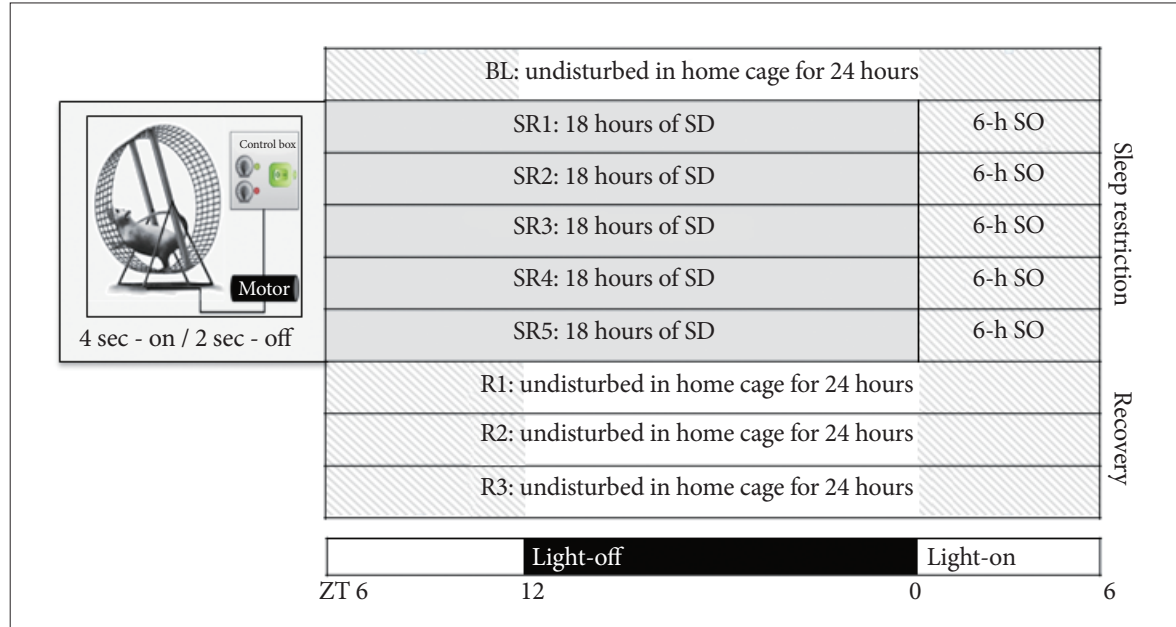

Fig. 1. Experimental design of chronic sleep restriction. Experiment schedule consists of 9 days: 1 baseline day (BL), 5 days of sleep restriction (SR), and 3 days of recovery $(R)$. Mouse was not disturbed during 6 hours of sleep opportunity (SO), 24 hours of $\mathrm{BL}$, and $\mathrm{R}$ days. Sleep was deprived by 4-s on and 2-s off schedule of automatic wheel. Shaded area represents SO in case of SR days and mouse sleep time on BL and $R$ days. ZT: zeitgeber time, SD: sleep deprivation.

Fig. 2. Examples of manually detected sleep structure. Recorded channels are described in left to figure. Frontal, signal from a screw electrode in frontal area; Parietal, signal from a screw electrode in parietal area; ACC, accelerometer signal for detecting body movements. $N R, R$, and $W$ in the middle of each 10 -s epoch indicates states manually scored. NR: non-rapid eye movement, REM: rapid eye movement, W: wake. 

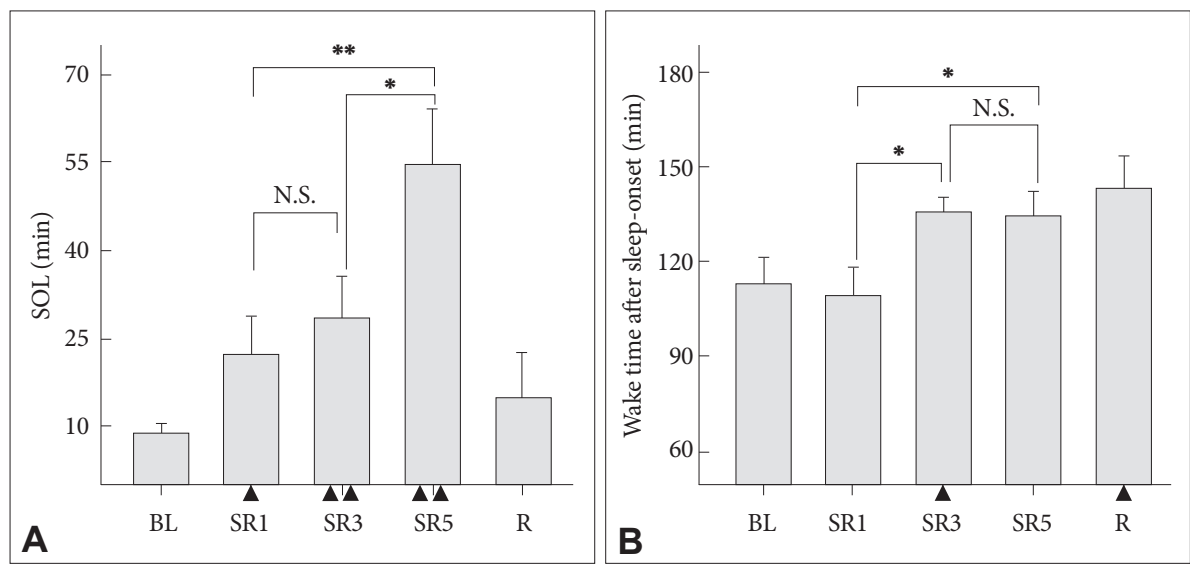

Fig. 3. Sleep-onset latency (SOL) and wake time after sleep-onset. A: Change of SOL as sleep deprivation continues. B: Comparison of wake time after sleeponset. The asterisk indicates statistical significance $\left({ }^{*} p<0.05 .{ }^{* *} p<0.01\right)$. The filled triangle below the bar indicates statistical significance compared to the baseline $(\boldsymbol{\Delta} p<0.05, \boldsymbol{\Delta} \Delta p<0.01)$. BL: baseline, N.S.: not significant, R: recovery, SR: sleep restriction.

Table 1. TWT and SOL, $\mathrm{n}=9$

\begin{tabular}{lrcccc}
\hline & Baseline & SR1 & SR3 & SR5 & Recovery \\
\hline TWT (min) & $122.7 \pm 8.8$ & $132.4 \pm 10.1$ & $164.6 \pm 4.7^{* *}$ & $189.4 \pm 7.9^{* *}$ & $158.7 \pm 13.8$ \\
SOL (min) & $8.6 \pm 2.0$ & $22.3 \pm 6.4^{*}$ & $28.1 \pm 7.4^{* *}$ & $54.6 \pm 9.6^{* *}$ & $14.9 \pm 7.6$ \\
\hline
\end{tabular}

Mean \pm standard error mean.

${ }^{*} \mathrm{p}<0.05 .{ }^{* *} \mathrm{p}<0.01$ (Paired t-test, compared to baseline).

TWT: total wake time, SOL: sleep onset latency.

ter paradigm, which is less stressful, but we observed the opposite result. In applying the motorized wheel paradigm, the animals experience a handling prior to sleep opportunity, which is different condition from the baseline sleep. Although the animals were given the sleep opportunity after sleep restriction at the same zeitgeber hour as baseline sleep, we cannot rule out the possibility of intervention effect in understanding the increase of SOL values. To avoid the handling issue, the handling of the animals should have been given to the baseline sleep around ZT0. However, this handling procedure does not explain the significant increase of SOL during sleep restriction days.

Besides, we cannot exclude the possibility that the sensitivity to stress in mice is higher than in rats. However, SOL increased gradually as sleep restriction continued, implying that an increase of SOL is not merely the effect of stress. In addition, the similar amount of time spent awake after sleep onset has been initiated compared to baseline indicates that the increased sleep pressure does not cause the mice to sleep for longer. Considering that the sleep debt is accumulated as sleep restriction continues, these results are difficult to understand unless there are other mechanisms, such as local sleep during awake time, ${ }^{19}$ that are present in mice. Or it may be that the relation between SOL and sleep pressure does not hold in mice.

\section{Acknowledgments}

This work was performed at the Center for Neuroscience, Korea Institute of Science and Technology (KIST), Seoul, South Korea.

This research was supported by: the KIST Institutional Programs 2E24480 (BK, JHC) and 2Z03990 (EH, JHC).

\section{Conflicts of Interest}

The authors have no financial conflicts of interest.

\section{REFERENCES}

1. Heslop P, Smith GD, Metcalfe C, Macleod J, Hart C. Sleep duration and mortality: the effect of short or long sleep duration on cardiovascular and all-cause mortality in working men and women. Sleep Med 2002;3:305-14.

2. Kripke DF, Garfinkel L, Wingard DL, Klauber MR, Marler MR. Mortality associated with sleep duration and insomnia. Arch Gen Psychiatry 2002;59:131-6.

3. Mesas AE, López-García E, León-Muñoz LM, Guallar-Castillón P, Rodríguez-Artalejo F. Sleep duration and mortality according to health status in older adults. J Am Geriatr Soc 2010;58:1870-7.

4. Belenky G, Wesensten NJ, Thorne DR, Thomas ML, Sing HC, Redmond DP, et al. Patterns of performance degradation and restoration during sleep restriction and subsequent recovery: a sleep dose-response study. J Sleep Res 2003;12:1-12.

5. Van Dongen HP, Maislin G, Mullington JM, Dinges DF. The cumulative cost of additional wakefulness: dose-response effects on neurobehavioral functions and sleep physiology from chronic sleep restriction and total sleep deprivation. Sleep 2003;26:117-26.

6. U.S. Bureau of Labor Statistics and U.S. Census Bureau. American Time Use Survey User's Guide - Understanding ATUS 2003 to 2013 (2013 June). 2007 [cited 2007 Mar 13]. Available from: http://www.bls. gov/tus/atususersguide.pdf.

7. Van Dongen H, Rogers NL, Dinges DF. Sleep debt: theoretical and empirical issues. Sleep Biol Rhythms 2003;1:5-13.

8. Horne JA, Wilkinson S. Chronic sleep reduction: daytime vigilance performance and EEG measures of sleepiness, with particular reference to "practice" effects. Psychophysiology 1985;22:69-78.

9. Youngstedt SD, Kline CE, Zielinski MR, Kripke DF, Devlin TM, Bogan $\mathrm{RK}$, et al. Tolerance of chronic 90-minute time-in-bed restriction in older long sleepers. Sleep 2009;32:1467-79.

10. Zielinski MR, Kline CE, Kripke DF, Bogan RK, Youngstedt SD. No effect of 8-week time in bed restriction on glucose tolerance in older long sleepers. J Sleep Res 2008;17:412-9.

11. Grønli J, Soulé J, Bramham CR. Sleep and protein synthesis-dependent synaptic plasticity: impacts of sleep loss and stress. Front Behav Neurosci $2014 ; 7: 224$. 
12. Carskadon MA, Dement WC. Cumulative effects of sleep restriction on daytime sleepiness. Psychophysiology 1981;18:107-13.

13. Carskadon MA, Dement WC, Mitler MM, Roth T, Westbrook PR, Keenan S. Guidelines for the multiple sleep latency test (MSLT): a standard measure of sleepiness. Sleep 1986;9:519-24.

14. Kim Y, Bolortuya Y, Chen L, Basheer R, McCarley RW, Strecker RE. Decoupling of sleepiness from sleep time and intensity during chronic sleep restriction: evidence for a role of the adenosine system. Sleep 2012; 35:861-9.

15. Everson CA, Bergmann BM, Rechtschaffen A. Sleep deprivation in the rat: III. Total sleep deprivation. Sleep 1989;12:13-21.
16. Manetto V, Medori R, Cortelli P, Montagna P, Tinuper P, Baruzzi A, et al. Fatal familial insomnia: clinical and pathologic study of five new cases. Neurology 1992;42:312-9.

17. Benedetti F, Fresi F, Maccioni P, Smeraldi E. Behavioural sensitization to repeated sleep deprivation in a mice model of mania. Behav Brain Res 2008;187:221-7.

18. Gessa GL, Pani L, Fadda P, Fratta W. Sleep deprivation in the rat: an animal model of mania. Eur Neuropsychopharmacol 1995;5 Suppl:89-93.

19. Vyazovskiy VV, Olcese U, Hanlon EC, Nir Y, Cirelli C, Tononi G. Local sleep in awake rats. Nature 2011;472:443-7. 